\title{
Disposición para participar en investigación en población adicta: prevalencia y factores asociados
}

\section{Willingness of patients with SUD to participate in research: prevalence and associated factors}

\author{
Inés Morán -Sánchez*, Aurelio Luna**, María D. Pérez-Cárcelees**. \\ * Centro de Salud Mental Cartagena, Servicio Murciano de Salud. Cartagena (Murcia), España; ** Departamento de Medicina \\ Legal y Forense. Biomedical Research Institute (IMIB), Regional Campus of International Excellence "Campus Mare \\ Nostrum", Facultad de Medicina, Universidad de Murcia, España.
}

\section{Resumen}

Cada vez se presta más atención a las motivaciones de las personas reclutadas para ensayos clínicos, especialmente si pertenecen a colectivos vulnerables. Aunque la participación en investigación de las personas con trastorno por uso de sustancias (TUS) suscita estereotipos negativos, muy pocos estudios se han centrado en los factores que influyen en la misma. Nuestro objetivo es analizar sus motivaciones comparando las razones y la disposición a participar en un ensayo hipotético de 53 pacientes con diagnósticos DSM- 5 de TUS y 50 controles. Las respuestas que dieron a la entrevista MacArthur Competence Assessment Tool for Clinical Research se correlacionaron con diversas variables. No encontramos diferencias significativas entre ambas poblaciones en términos de motivaciones y disposición a participar. El $59 \%$ de la población TUS mencionó altruismo, un $53,8 \%$ esperaba beneficio terapéutico, y el $43,6 \%$ deseaba ayudar a otros. De los pacientes con TUS que rechazaron participar, el $69,2 \%$ alegó miedo y el $46,2 \%$ incomodidad por los riesgos. La disposición a participar se relacionó con un mayor nivel cognitivo y de alfabetización informática. En el análisis multivariante, la aversión a la investigación permaneció como factor predictivo significativo de la disposición a participar. Cuando la investigación no está relacionada con su diagnóstico, las motivaciones de la población TUS son similares a las de los controles y se deducen lógicamente del estudio, aunque también se evidenciaron elementos de "error terapéutico". Por consiguiente, las visiones negativas sobre las motivaciones de los TUS como participantes en investigación son infundadas. Para mejorar el reclutamiento, las valoraciones deben dirigirse a vulnerabilidades especificas en lugar de al diagnóstico. Palabras clave: Adicciones; Ética en investigación; Motivación; Toma de decisiones.

\begin{abstract}
Greater attention is focusing on the motivations of subjects recruited for research protocols, especially in vulnerable populations. Although addiction is a highly stigmatized condition, very little research has focused on the factors influencing the decision to participate of patients with an addiction. Our aim is to gather further evidence in relation to the motivations of people with Substance Use Disorders (SUD), comparing their reasoning and willingness to participate in a hypothetical research study of 53 subjects with DSM- 5 diagnoses of SUD and 50 controls. Responses on the MacArthur Competence Assessment Tool for Clinical Research were documented and correlated with several variables. There were no significant differences in willingness to participate in research and reasons for doing so between SUD and controls. Among SUD subjects, 59\% mentioned altruism, $53.8 \%$ expected therapeutic benefits, and $43.6 \%$ desired to help others; none mentioned money. Of those patients with SUD who refused to participate in research, $69.2 \%$ cited aversion and $46.2 \%$ mentioned risk. Willingness to participate was correlated with higher computer literacy and better cognitive performance. In the multivariate analysis, aversion was a significant predictor of willingness to participate in research. When research is not related to their diagnosis, the motivations of SUD and controls are similar and flowed logically from the study. However, elements associated with therapeutic misconceptions were also evident. Therefore, negative views about the motivations of SUD subjects' participation in research are unfounded. Consequently, to improve study recruitment, assessments may be targeted to specific vulnerabilities rather than to diagnoses.

Keywords: Addictions; Research ethics; Motivation; Decision-making.
\end{abstract}

Recibido: Abril 2017; Aceptado: Noviembre 2017.

Enviar correspondencia a:

Inés Morán Sánchez. CSM Cartagena. Calle Real, 8. CP 30201, Cartagena (Murcia), España.

E-mail: ines.moran@carm.es 
$\mathbf{P}$ ara el avance del conocimiento científico y la mejora de la atención a los pacientes, es imprescindible la investigación biomédica. El éxito de la misma depende de una tasa de reclutamiento y retención adecuada de las personas que participan. En los últimos años, se está prestando interés al estudio de las motivaciones y la disposición a participar en investigación (Geppert, Candilis, Baker, Lidz y Appelbaum, 2014; Lawton et al., 2016; Tromp, Zwaan y van de Vathorst, 2016), sobre todo en poblaciones vulnerables o marginales (Barrat, Norman y Fry, 2007; Candilis, Geppert, Fletcher, Lidz y Appelbaum, 2006). Aunque la adicción es una condición estigmatizada que suscita dudas sobre su motivación real para contribuir al avance científico, si revisamos los ámbitos de investigación en adicciones (Nogué y Miró, 2015), muy poca se ha centrado en los factores que influyen en la participación (Barrat et al., 2007; Fry y Dwyer, 2001).

Estudios previos en población general han explorado las razones que esgrime el participante en investigación: acceso a la información, ganancia económica, curiosidad, deseo de ayudar a otros y contribuir a la ciencia (Candilis et al., 2006; Seelig y Dobelle, 2001). También hay factores negativos, como miedo e incomodidad con los procedimientos, que pueden servir como barreras para la participación o reducir la adherencia en los estudios (Ammassari et al., 2002; Brintnall-Karabelas et al., 2011). Desconocemos hasta qué grado se pueden extrapolar a la población diagnosticada de TUS estos hallazgos. Los pocos estudios que hay sobre población adicta en esta área, exploran el papel de los incentivos económicos en investigación (Barrat et al., 2007; Fry et al., 2001). Algunos argumentan que desde una perspectiva ética, recibir dinero por participar en investigación, podría invalidar el consentimiento informado (Fry, Hall, Ritter y Jenkinson, 2006a; Misra, Socherman, Park, Hauser y Ganzini, 2008). Los sujetos podrían asumir riesgos que si no fuera por el incentivo no asumirían, lo que invalidaría el principio de justicia al condicionar más a personas en situación de desventaja socioeconómica, como son los TUS (Carter y Hall, 2012; Dunn, Kim, Fellows y Palmer, 2009).

Otra razón, para tratar de comprender mejor los motivos de la población con TUS para participar en investigación, se relaciona con la prevalencia de estereotipos negativos sobre la adicción (Morera, 2000) y las suposiciones que se tienen sobre este colectivo incluso desde los mismos profesionales que les tratan (Barrat et al., 2007).

Hasta lo que sabemos, no existe ningún estudio en población española que revise los factores que animan o desalientan a los pacientes con TUS a participar en investigación. Conocer las motivaciones de esta población, permitiría a los profesionales saber a qué aspectos los pacientes les dan más importancia y qué información es relevante para tomar su decisión. Esto permitiría diseñar el proceso de reclutamiento y consentimiento informado desde la perspectiva y las necesidades de la población adicta. El objetivo de este estudio es proporcionar mayor evidencia sobre la cuestión de por qué los sujetos TUS participan en investigaciones en nuestro medio.

\section{Método}

\section{Tipo de estudio}

Se realizó un estudio transversal aprobado por el Comité Ético de Investigación de nuestro hospital de referencia (Complejo Hospitalario Universitario de Cartagena).

\section{Participantes}

Este estudio deriva de otro que compara la capacidad para participar en investigación en población adicta. Se pueden consultar los detalles completos en otra parte (Morán-Sánchez, Luna, Sánchez, Aguilar y Pérez-Cárceles, 2016). La investigación actual se centra en las motivaciones y la disposición a participar de 53 pacientes tratados por consumo de alcohol y/o sustancias ilícitas en un Centro de Atención al Drogodependiente y 50 controles sin patología psiquiátrica de un Centro de Salud. Se invitó a participar a todos los pacientes que acudieran consecutivamente durante un período de 4 meses a los centros de estudio. Los participantes incluyeron pacientes ambulatorios con diagnósticos DSM-5 de TUS y controles diagnosticados de hipertensión, diabetes mellitus u otras enfermedades crónicas. Los criterios de inclusión fueron: (a) edad actual de 18 años o más, (b) diagnóstico de las patologías diana del estudio, (c) hablar español fluido, (d) puntuación de $20 \mathrm{o}$ más en la versión española del Mini-Mental State Examination: MEC (Lobo et al., 1999), y (e) otorgar consentimiento voluntario.

Los controles se excluyeron si (a) cumplían criterios actuales para un TUS u otros diagnósticos DSM-5 (American Psychiatric Association, 2014), (b) eran pacientes activos del Centro de Salud Mental o del Centro de Atención al Drogodependiente o (c) estaban en tratamiento psiquiátrico con su médico de familia.

Los consumidores fueron excluidos si estaban intoxicados o sufriendo síndrome de abstinencia cuando les pedíamos el consentimiento.

\section{Medidas}

La información de los participantes se recogió mediante un cuestionario diseñado para obtener variables demográficas y clínicas. El nivel de funcionamiento de la población adicta se evaluó utilizando la Escala de Evaluación de la Actividad Global (Endicott, Spitzer, Fleiss y Cohen, 1976), y la gravedad de sus síntomas se evaluó con la Escala de Impresión Clínica Global (Guy, 1976).

Las motivaciones y la disposición a participar en investigación se recogieron de las respuestas obtenidas en la versión española de la escala MacArthur Competence As- 
sessment Tool for Clinical Research (MacCAT-CR) (Baón, 2013). Este instrumento es una entrevista semi-estructurada que se adapta a los elementos de un protocolo concreto de investigación y evalúa las 4 dimensiones más reconocidas de la capacidad de toma de decisiones: (a) comprensión de la información; (b) apreciación de las consecuencias en las circunstancias concretas de cada paciente; (c) razonamiento sobre decidir participar o no y $(\mathrm{d})$ expresión de una elección sobre la participación en el proyecto (Appelbaum y Grisso, 2001). La aplicación de la entrevista MacCAT-CR implica dar información sobre el estudio en el que se pide a los sujetos considerar participar, seguido de preguntas que evalúan la capacidad y se puntúan de 0-2, reflejando las puntuaciones más altas un mejor rendimiento. La escala de Comprensión tiene trece preguntas, la de Apreciación tiene tres, la escala de Razonamiento cuatro preguntas y la escala de Expresión de una elección sólo una. Este instrumento ha sido ampliamente utilizado en investigación y se describe más detalladamente en otra parte (Appelbaum et al., 2001). La disposición de los sujetos a participar en el estudio hipotético se obtuvo de las respuestas a la subescala Expresión de una elección ( "Ahora que ha tenido más tiempo para pensarlo, me gustaría preguntarle nuevamente si piensa que es más probable que participara /no participara en este estudio'). Las motivaciones para participar en investigación se obtuvieron a partir de las respuestas a la subescala Razonamiento ( "Así que piensa que elegiría estar / no estar en el estudio. ¿Qué es lo que lo convierte en la mejor opción para usted?") que se recogieron y codificaron según su contenido.

El consentimiento hipotético que diseñamos describía un ensayo clínico aleatorizado controlado con placebo de un compuesto experimental para tratar el dolor de cabeza. El formulario describía extracción de sangre y efectos secundarios sin riesgo vital. También se informaba del carácter voluntario de la participación, la incapacidad para garantizar un beneficio directo y la posibilidad de retirada.

\section{Procedimientos}

Una vez que se obtenía el consentimiento informado por escrito, se valoraba el nivel cognitivo con el MEC-30 excluyendo a aquellos participantes con un deterioro avanzado. Posteriormente, se leía el proyecto hipotético en voz alta y se administraba la entrevista MacCAT-CR que se puntuaba según los criterios de su manual.

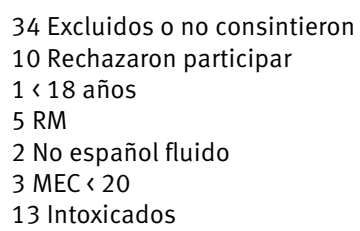

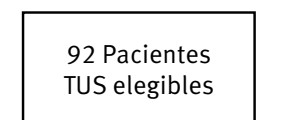

6 No disponibles

5 Pérdidas

1 No completó

MacCAT-CR

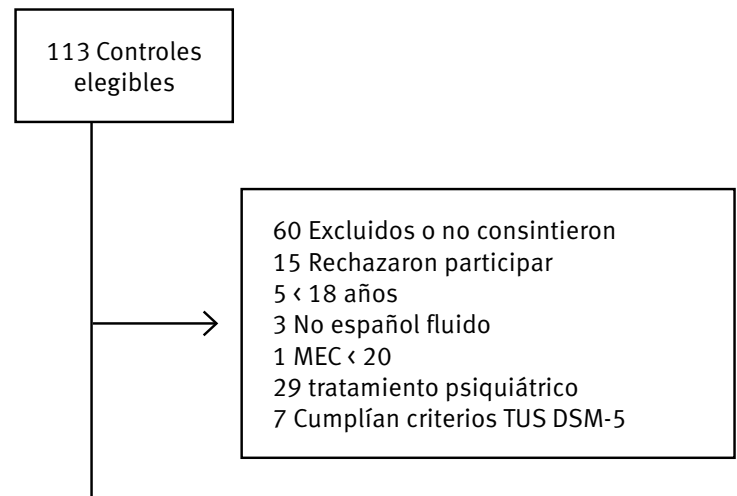

53 Controle evaluados

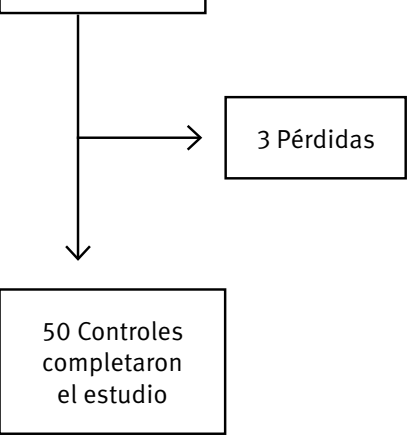

Figura 1. Flujo de inclusión de los participantes

Nota. TUS: Trastorno por Uso de Sustancias; MEC: Mini Examen Cognoscitivo; RM: retraso mental; MacCAT-CR: MacArthur Competence Assessment Tool for Clinical Research 
Tabla 1. Características basales de los participantes en el estudio

\begin{tabular}{|c|c|c|c|}
\hline & $\begin{array}{r}\text { TUS } \\
(n=53)\end{array}$ & $\begin{array}{l}\text { Control } \\
(n=50)\end{array}$ & $\mathbf{p}$ \\
\hline Edad (Media en años - DT-) & $42.9(11.9)$ & $48.6(11.9)$ & $.037^{\mathrm{b}}$ \\
\hline Mujeres (\%) & 28.3 & 62 & $<.001^{\mathrm{a}}$ \\
\hline Estado civil (\%) & & & $<.001^{\mathrm{a}}$ \\
\hline $\begin{array}{l}\text { Casado/conviviendo } \\
\text { Nunca casado } \\
\text { Anteriormente casado }\end{array}$ & $\begin{array}{l}32.1 \\
41.5 \\
26.4\end{array}$ & $\begin{array}{r}82 \\
6 \\
12\end{array}$ & \\
\hline Tipo de convivencia (\%) & & & $<.001^{c}$ \\
\hline $\begin{array}{l}\text { Solo } \\
\text { Con familia propia } \\
\text { Con familia de origen/institución }\end{array}$ & $\begin{array}{l}15.1 \\
45.3 \\
39.6\end{array}$ & $\begin{array}{l}12 \\
88\end{array}$ & \\
\hline Nivel de estudios (\%) & & & $<.001^{\mathrm{a}}$ \\
\hline $\begin{array}{l}\text { Primarios } \\
\text { Secundarios } \\
\text { Estudios universitarios }\end{array}$ & $\begin{array}{l}58.2 \\
32.1 \\
9.45\end{array}$ & $\begin{array}{l}20 \\
20 \\
40\end{array}$ & \\
\hline Situación laboral (\%) & & & $<.001^{\circ}$ \\
\hline $\begin{array}{l}\text { Activo } \\
\text { Desempleado } \\
\text { Jubilado } \\
\text { Discapacitado }\end{array}$ & $\begin{array}{l}20.8 \\
45.3 \\
13.2 \\
20.8\end{array}$ & $\begin{array}{r}84 \\
2 \\
14\end{array}$ & \\
\hline Investigación previa (\%) & & & $<.001^{\mathrm{a}}$ \\
\hline $\begin{array}{l}\text { Sí } \\
\text { No }\end{array}$ & $\begin{array}{r}1.9 \\
98.1\end{array}$ & $\begin{array}{l}46 \\
54\end{array}$ & \\
\hline Alfabetización informática (\%) & & & $<.001^{\mathrm{a}}$ \\
\hline $\begin{array}{l}\text { Sí } \\
\text { No }\end{array}$ & $\begin{array}{l}81.1 \\
18.9 \\
\end{array}$ & $\begin{array}{r}96 \\
4 \\
\end{array}$ & \\
\hline $\begin{array}{l}\text { MEC (rango, 0-30) (Media en } \\
\text { puntos -DT-) }\end{array}$ & $28.2(4.2)$ & $29.6(0.9)$ & $<.001^{b}$ \\
\hline
\end{tabular}

Nota. DT: desviación típica; TUS: Trastorno por Uso de Sustancias; MEC: Mini Examen Cognoscitivo. ${ }^{\mathrm{a}} \chi 2$ de Pearson; ${ }^{\mathrm{b}} \mathrm{U}$ Mann-Whitney; ${ }^{\mathrm{C} E s t a d i ́ s t i c o ~ d e ~ F i s h e r . ~}$

\section{Análisis estadístico}

Los datos fueron analizados con el programa SPSS (versión 19.0). Previó al análisis se comprobó la distribución de las variables continuas para ver si cumplían criterios de normalidad. Las diferencias en los datos ordinales o continuos se analizaron mediante el test de U de Mann-Whitney. Para las diferencias entre las variables categóricas, usamos la $\chi 2$ de Pearson o el test exacto de Fisher para los datos no paramétricos.

Para identificar los factores predictores de la disposición a participar en investigación, realizamos un análisis de regresión logística calculando los Odds Ratios (OR) y sus IC al 95\%. Se incluyeron en el análisis multivariante aquellas variables independientes con significación estadística en el análisis univariante y aquellas clínicamente relevantes aunque no alcanzaran significación. El tamaño muestral del presente estudio fue el máximo que se pudo conseguir durante el periodo de reclutamiento. Se podría considerar suficiente para el modelo de regresión logística según la fórmula de cálculo propuesta por Peduzzi de al menos 10 casos en cada uno de los valores posibles de la variable respuesta (Ortega y Cayuela, 2002). Según se recomienda en la literatura, utilizamos el método de introducción directa de las variables para obtener un modelo con las variables directamente relacionadas con la variable dependiente (Aguayo, 2010; Nuñez, Steyerberg y Nuñez, 2011).

Valoramos la validez del modelo con la prueba ómnibus sobre los coeficientes, las R cuadrado de Cox y Snell y de Nagelkerke y el test de Hosmer-Lemeshow. Para todos los análisis se utilizó un nivel de significación de .05.

\section{Resultados}

De los 205 sujetos elegibles para este estudio, 103 fueron excluidos o no estaban disponibles para participar por varias razones. El diagrama de flujo de pacientes durante el estudio se recoge en la Figura 1.

Las características basales de los participantes en el estudio se exponen en la Tabla 1.

Los 53 pacientes con TUS tenían los siguientes diagnósticos (no mostrado): $45.3 \%(n=24)$ tenían un trastorno por consumo de alcohol o cannabis, el $18.9 \%(n=10)$ consumían cocaína y un 35.8\% $(n=19)$ consumía alcohol y otra droga. Los pacientes que consumían THC eran más jóvenes 32.92 años $(D T=12.72)$, y los que consumían alcohol mayores 51.67 años $(D T=7.63) ; \chi 2(2, \mathrm{~N}=53)=11.81$, $\mathrm{p}=.003$. La duración de la enfermedad también fue mayor en el grupo de alcohol $19.67(D T=13.26)$ que en el resto de grupos (7.42 años $(D T=7.13)$ en los consumidores de THC; 9.80 años $(D T=6.56)$ en los consumidores de cocaína y 18.11 años $(D T=9.13)$ en los que consumían alcohol y otra sustancia; $\chi 2(2, \mathrm{~N}=53)=6.45, \mathrm{p}=.04)$. En el resto de variables estudiadas no hubo diferencias estadísticamente significativas.

\section{Disposición a participar en investigación y factores relacionados.}

El 75\% $(n=39)$ del grupo TUS y el 78\% $(n=39)$ del grupo control estaban dispuestos a participar en el proyecto hipotético de investigación, no existiendo diferencias estadísticamente significativas entre ambos grupos, $\chi 2$ (1, $\mathrm{N}=102)=7.21, \mathrm{p}=.721$.

Las características de los pacientes TUS en función de su disposición a participar en la investigación hipotética, se describen en la Tabla 2. Encontramos diferencias significativas en las puntuaciones MEC $(\mathrm{Z}=-1.99, \mathrm{p}=.047)$ y en el grado de alfabetización informática $(87 \%$ grado alto de alfabetización informática entre los dispuestos a participar vs $61.5 \%$ entre los no dispuestos; $\chi 2(1, \mathrm{~N}=52)=4.13$, $\mathrm{p}$ $=.042$ ). En el resto de variables estudiadas (sociodemográficas, clínicas y de capacidad de toma de decisiones), no hubo diferencias estadísticamente significativas entre los dispuestos a participar y los no dispuestos. 
Tabla 2. Características de la población TUS según su disposición a participar en investigación

\begin{tabular}{|c|c|c|c|}
\hline & \multicolumn{2}{|c|}{ Disposición a participar } & \multirow[t]{2}{*}{$\mathbf{p}$} \\
\hline & Sí (n=39) & No $(n=13)$ & \\
\hline Edad (Media en años - DT-) & $44.1(11.1)$ & 40.5 (13.9) & $.533^{c}$ \\
\hline Mujeres (\%) & 35.9 & 7.7 & $.078^{\mathrm{b}}$ \\
\hline Estado civil (\%) & & & $.614^{\mathrm{b}}$ \\
\hline $\begin{array}{l}\text { Casado/conviviendo } \\
\text { Nunca casado } \\
\text { Anteriormente casado }\end{array}$ & $\begin{array}{l}33.3 \\
38.5 \\
28.2\end{array}$ & $\begin{array}{l}23.1 \\
53.8 \\
23.1\end{array}$ & \\
\hline Tipo de convivencia (\%) & & & $.641^{\mathrm{b}}$ \\
\hline $\begin{array}{l}\text { Solo } \\
\text { Con familia propia } \\
\text { Con familia de origen/institución }\end{array}$ & $\begin{array}{l}12.8 \\
48.7 \\
38.5\end{array}$ & $\begin{array}{l}16.7 \\
62.5 \\
20.8\end{array}$ & \\
\hline Nivel de estudios (\%) & & & $.263^{b}$ \\
\hline $\begin{array}{l}\text { Primarios } \\
\text { Secundarios } \\
\text { Estudios universitarios }\end{array}$ & $\begin{array}{c}59 \\
28.2 \\
12.8\end{array}$ & $\begin{array}{l}53.8 \\
46.2\end{array}$ & \\
\hline Situación laboral (\%) & & & $.947^{\mathrm{b}}$ \\
\hline $\begin{array}{l}\text { Activo } \\
\text { Desempleado } \\
\text { Jubilado } \\
\text { Discapacitado }\end{array}$ & $\begin{array}{l}23.1 \\
43.6 \\
12.8 \\
20.5\end{array}$ & $\begin{array}{l}15.4 \\
46.2 \\
15.4 \\
23.1\end{array}$ & \\
\hline Investigación previa (\%) & & & $1.00^{\mathrm{b}}$ \\
\hline $\begin{array}{l}\text { Sí } \\
\text { No }\end{array}$ & $\begin{array}{c}2.6 \\
97.4\end{array}$ & 100 & \\
\hline Alfabetización informática (\%) & & & $.042^{\mathrm{b}}$ \\
\hline $\begin{array}{l}\text { Sí } \\
\text { No }\end{array}$ & $\begin{array}{l}87.2 \\
12.8\end{array}$ & $\begin{array}{l}61.5 \\
38.5\end{array}$ & \\
\hline Diagnóstico psiquiátrico (\%) & & & $.166^{c}$ \\
\hline ICG (\%) & & & $1.00^{\mathrm{a}}$ \\
\hline $\begin{array}{l}\leq \text { Moderadamente enfermo } \\
\geq \text { Moderadamente enfermo }\end{array}$ & $\begin{array}{l}23.1 \\
76.9\end{array}$ & $\begin{array}{l}23.1 \\
76.9\end{array}$ & \\
\hline EEAG (rango, 0-100) (Media en puntos -DT-) & $67.1(15.1)$ & $63.1(17.0)$ & $.444^{\mathrm{b}}$ \\
\hline Duración de la enfermedad (Media en años - DT-) & $14.9(10.3)$ & $14.2(11.5)$ & $.734^{\mathrm{b}}$ \\
\hline Hospitalizaciones psiquiátricas (Media en unidades - DT-) & $1.1(2.4)$ & $1.1(1.9)$ & $.981^{\mathrm{b}}$ \\
\hline Ingresos en comunidades terapéuticas (Media en unidades -DT-) & $0.47(0.9)$ & $0.23(0.4)$ & $.571^{\mathrm{b}}$ \\
\hline MEC (rango, 0-30) (Media en puntos -DT-) & $28.5(1.6)$ & $29.5(0.8)$ & $.047^{\mathrm{b}}$ \\
\hline Grupo (\%) & & & $.593^{c}$ \\
\hline $\begin{array}{l}\text { Alcohol } \\
\text { THC } \\
\text { Cocaína } \\
\text { Alcohol + otro }\end{array}$ & $\begin{array}{l}23.1 \\
15.4 \\
25.6 \\
35.9\end{array}$ & $\begin{array}{l}23.1 \\
38.5 \\
38.5\end{array}$ & \\
\hline \multicolumn{4}{|l|}{ MacCAT-CR (Media en puntos -DT-) } \\
\hline $\begin{array}{l}\text { Puntuación Comprensión (rango, 0-26) } \\
\text { Puntuación Apreciación (rango, 0-6) } \\
\text { Puntuación Razonamiento (rango, 0-8) }\end{array}$ & $\begin{array}{l}20.9(4.2) \\
5.1(1.3) \\
6.3(1.5)\end{array}$ & $\begin{array}{l}19(5.0) \\
5.1(0.7) \\
5.9(1.9)\end{array}$ & $\begin{array}{l}.840^{\mathrm{b}} \\
.549^{\mathrm{b}} \\
.178^{\mathrm{b}}\end{array}$ \\
\hline Expresión de una elección (\%) & & & $.672^{\mathrm{c}}$ \\
\hline $\begin{array}{l}2 \\
1 \\
0\end{array}$ & $\begin{array}{c}92.3 \\
5.1 \\
2.6\end{array}$ & $\begin{array}{l}84.6 \\
15.4\end{array}$ & \\
\hline Capacidad (\%) & & & $.735^{c}$ \\
\hline $\begin{array}{l}\text { Sí } \\
\text { No }\end{array}$ & $\begin{array}{l}69.2 \\
30.8\end{array}$ & $\begin{array}{l}61.5 \\
38.5\end{array}$ & \\
\hline
\end{tabular}

Nota. DT: desviación típica; ICG: Escala de Impresión Clinical Global; EEAG: Escala de Evaluación de la Actividad Global; THC: Tetra hidro cannabinol; MEC: Mini

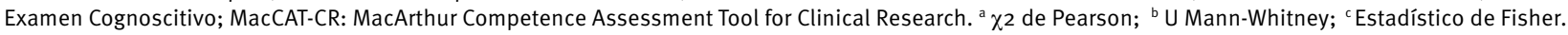


Tabla 3. Motivaciones para participar o no en investigación

\begin{tabular}{|c|c|c|c|}
\hline & Control & TUS & $\mathbf{p}$ \\
\hline \multicolumn{4}{|l|}{ Razones para aceptar participar (\%) } \\
\hline $\begin{array}{l}\text { Altruismo/deseo de avance de la ciencia } \\
\text { Expectativa de un beneficio propio } \\
\text { Deseo de ayudar a otros } \\
\text { Otros motivos distintos a los anteriores }\end{array}$ & $\begin{array}{c}59(n=23) \\
46.2(n=18) \\
48.7(n=19) \\
20.5(n=8)\end{array}$ & $\begin{array}{c}59(n=23) \\
53.8(n=21) \\
43.6(n=17) \\
17.9(n=7)\end{array}$ & $\begin{array}{l}1.00^{\mathrm{a}} \\
.497^{\mathrm{a}} \\
.650^{\mathrm{a}} \\
.774^{\mathrm{a}}\end{array}$ \\
\hline \multicolumn{4}{|l|}{ Razones para rechazar participar (\%) } \\
\hline $\begin{array}{l}\text { Aversión a la investigación } \\
\text { Incomodidad por los efectos secundarios } \\
\text { Otros motivos distintos a los anteriores }\end{array}$ & $\begin{array}{c}90.9(n=10) \\
36.4(n=4) \\
9.1(n=1)\end{array}$ & $\begin{array}{l}69.2(n=9) \\
46.2(n=6) \\
15.4(n=2)\end{array}$ & $\begin{array}{l}.327^{\mathrm{b}} \\
.697^{\mathrm{b}} \\
1.00^{\mathrm{b}}\end{array}$ \\
\hline
\end{tabular}

Nota. TUS: Trastorno por Uso de Sustancias. ${ }^{\mathrm{a}} \chi 2$ de Pearson. ${ }^{\mathrm{b}}$ Estadístico de Fisher.

Tabla 4. Motivaciones para participar en investigación según disposición a participar y grupo

\begin{tabular}{|c|c|c|c|c|c|c|c|}
\hline & \multirow{2}{*}{$\begin{array}{l}\text { Disposición a } \\
\text { participar }\end{array}$} & \multicolumn{3}{|c|}{ Control } & \multicolumn{3}{|c|}{ TUS } \\
\hline & & $\%$ & OR IC $95 \%$ & $\mathrm{p}$ & $\%$ & OR IC $95 \%$ & $\mathrm{p}$ \\
\hline Altruismo & $\begin{array}{l}\text { Participa } \\
\text { No participa }\end{array}$ & $\begin{array}{c}59 \\
0\end{array}$ & $1.69(1.23-2.31)$ & $<.001^{\mathrm{a}}$ & $\begin{array}{c}59 \\
23.1\end{array}$ & $4.79(1.14-20.21)$ & $0.25^{\mathrm{a}}$ \\
\hline Beneficio personal & $\begin{array}{l}\text { Participa } \\
\text { No participa }\end{array}$ & $\begin{array}{c}46.2 \\
0\end{array}$ & $1.52(1.19-1.96)$ & $.004^{\mathrm{b}}$ & $\begin{array}{l}53.8 \\
15.4\end{array}$ & $6.42(1.26-32.84)$ & $<.001^{\mathrm{a}}$ \\
\hline Ayudar a otros & $\begin{array}{l}\text { Participa } \\
\text { No participa }\end{array}$ & $\begin{array}{c}48.7 \\
9.1\end{array}$ & $9.5(1.11-81.51)$ & $.033^{\mathrm{b}}$ & $\begin{array}{l}43.6 \\
15.4\end{array}$ & $4.25(0.83-21.78)$ & $.099^{a}$ \\
\hline Aversión a la investigación & $\begin{array}{l}\text { Participa } \\
\text { No participa }\end{array}$ & $\begin{array}{c}2.6 \\
90.9\end{array}$ & $35.46(5.08-247.63)$ & $<.001^{\mathrm{b}}$ & $\begin{array}{c}5.1 \\
69.2\end{array}$ & $10.75(4.23-27.35)$ & $<.001^{b}$ \\
\hline Efectos secundarios & $\begin{array}{l}\text { Participa } \\
\text { No participa }\end{array}$ & $\begin{array}{c}5.1 \\
36.4\end{array}$ & $4.19(1.73-10.14)$ & $.017^{\mathrm{b}}$ & $\begin{array}{c}5.1 \\
46.2\end{array}$ & $6.57(3.32-13.00)$ & $.002^{\mathrm{b}}$ \\
\hline Motivos distintos & $\begin{array}{l}\text { Participa } \\
\text { No participa }\end{array}$ & $\begin{array}{c}20.5 \\
9.1\end{array}$ & $2.58(0.29-23.24)$ & $.662^{\mathrm{b}}$ & $\begin{array}{l}17.9 \\
15.4\end{array}$ & $1.20(0.22-6.69)$ & $.832^{\mathrm{b}}$ \\
\hline
\end{tabular}

Nota. IC: Intervalo de Confianza; OR: Odds Ratio; TUS: Trastorno por Uso de Sustancias. ${ }^{\mathrm{a}} \chi 2$ de Pearson. ${ }^{\mathrm{b}}$ Estadístico de Fisher.

\section{Motivaciones para participar en investigación.}

Las respuestas en la subescala MacCAT-CR de Razonamiento, que indaga sobre las motivaciones para decidir participar o no en un estudio, se clasificaron en seis categorías y se recogen en la Tabla 3:

a. Altruismo/deseo de avance de la ciencia

b. Expectativa de un beneficio propio

c. Deseo de ayudar a otros

d. Incomodidad por los efectos secundarios

e. Aversión a la investigación

f. Otros motivos distintos a los anteriores

El $59 \%(n=23)$ de la población adicta que se mostró dispuesta a participar en el estudio hipotético, mencionó altruismo como motivo, el $53.8 \%(n=21)$ expectativa de recibir un beneficio propio y un $43.6 \%(n=17)$ alegó el deseo de ayudar a otros. Mientras que en los pacientes TUS que decidieron no participar, el motivo más frecuente esgrimido fue la aversión a la investigación 69.2\% $(n=9)$ seguido de incomodidad por los efectos secundarios en un $46.2 \%(n=6)$. Como observamos en la Tabla 3, no encon- tramos diferencias significativas entre el grupo control y TUS en las motivaciones para participar en investigación clínica.

En la tabla 4 observamos que era aproximadamente 2 veces más probable que los controles participaran en investigación si mencionaban el altruismo que si no lo hacían (OR 1.69, IC 95\% 1.23 - 2.31; p <.001); mientras que en población TUS esta probabilidad era 5 veces mayor (OR 4.79, IC 95\% $1.14-20.21 ; \mathrm{p}=.025$ ). Aquellos sujetos TUS que adujeron recibir un mejor tratamiento tenían, aproximadamente, 7 veces más probabilidad de participar en la investigación (OR 6.42, IC 95\% 1.26 - 32.84; $\mathrm{p}=<.001$ ). Ninguno mencionó la posibilidad de recibir dinero como motivación para decidir participar.

Por el contrario, los que expresaron aversión a la investigación fueron más propensos a negarse a participar que los que no lo hicieron, (OR 10.75, IC 95\% 4.23-27.34; p < .001). En el grupo control esta probabilidad se elevó 35 veces más, (OR 35.46, IC 95\% 5.08-247.63; p <.001). La incomodidad por los efectos secundarios se asoció a una 
Tabla 5. Factores asociados a la disposición a participar en investigación

\begin{tabular}{lccc}
\hline Factores asociados & \multicolumn{1}{c}{ Análisis univariante } & Análisis multivariante $^{*}$ & OR IC 95\% \\
\hline MEC & OR IC 95\% & $\mathrm{p}$ & - \\
Alfabetización informática & $1.94(0.99-3.79)$ & .054 & - \\
Altruismo & $4.25(0.988-18.29)$ & .052 & - \\
Beneficio personal & $4.79(1.14-20.21)$ & 0.25 & - \\
Aversión por la investigación & $6.42(1.26-32.84)$ & $<.001$ & - \\
Efectos secundarios & $10.7584 .23-27.34) \cdot$ & $<.001$ & .029 \\
\hline
\end{tabular}

Nota. MEC: Mini Examen Cognoscitivo; OR: Odds Ratio; IC: Intervalo de Confianza; TUS: Trastorno por Uso de Sustancias. *Solo se muestran los factores con un valor de $p<.05$. Se muestra la OR recíproca cuando la $O R<1$.

probabilidad aproximadamente 7 veces mayor de no participar entre el grupo TUS (OR 6.57, IC 95\% 3.32-13.00; $\mathrm{p}$ $=.002)$.

En el análisis univariado todas las motivaciones esgrimidas se asociaron de forma significativa en ambos grupos con la disposición o no a participar excepto el dominio de "Otros motivos" y en el grupo TUS además, el dominio "Deseo de ayudar a otros" (Tabla 4). El modelo de regresión logística incluyó las variables relevantes significativamente asociadas con la disposición a participar en el análisis univariado y variables relevantes de limitación de la participación, (ver Tabla 5). Sólo una de las variables incluidas en el análisis univariante se mantuvo en el modelo multivariado: la aversión a la investigación (OR 14.24, IC 95 \% 1.31-154.8; p = $.028)$, es decir era 14 veces más probable que alguien no participe en investigación si tiene miedo.

El modelo resultó significativo $\chi 2(6, \mathrm{~N}=52)=29.61, \mathrm{p}$ $=.001$, explica entre el 43.4 y el $64.3 \%$ de la variable dependiente, y clasifica correctamente el $90.4 \%$ de los casos, por tanto es un modelo aceptable. En el test de Hosmer-Lemeshow obtuvimos un valor de p elevado que indica que la diferencia entre los valores observados y pronosticados fue pequeña, $\chi 2(8, \mathrm{~N}=52)=8.79, \mathrm{p}=.361$.

\section{Respuestas cualitativas}

La mayoría de los sujetos dispuestos a participar, expresaron motivos altruistas: querían "ayudar" a "la ciencia" o "a los médicos" o "contribuir a que salgan mejores medicamentos". Otros querían "mejorar el bienestar de las personas" o "de la sociedad".

El segundo motivo más frecuente esgrimido por los dispuestos a participar en ambos grupos, fue la expectativa de recibir un mejor tratamiento. Estos sujetos señalaron que participarían "por su beneficio personal”, "por si me encuentro mejor, porque tengo dolores" o "por arreglarme la cabeza, que no la tengo bien". Otros querían saber más sobre su dolor de cabeza "a ver si me miran bien y me dicen por qué me pasa esto" o "si me ven más médicos, podré saber más de lo mí". De los 53 sujetos con TUS, 13 expresaron a la vez motivos altruistas y personales.
Los sujetos que expresaron aversión a la investigación adujeron no participar "por miedo", por no ser "conejillos de indias que para eso, ya están los animales" o porque "los experimentos no son seguros". Algunos prefirieron no arriesgarse habiendo otros medicamentos ya contrastados. Tampoco querían tomar medicamentos nuevos: "porque pastillas que no conozco, no me las tomo". Otros mencionaron incomodidad por "tener que sacarse sangre", o manifestaron que no les gustaba tomar pastillas "por los efectos secundarios".

Algunos de los que expresaron otras razones dijeron que participarían porque confiaban en la entrevistadora: "porque me lo pides tú que me lo has explicado muy bien y eres muy agradable". Otros vieron en el proyecto la posibilidad de " $r$ lacionarse con gente" "compensar todo el mal que he hecho antes".

\section{Discusión}

Creemos que la importancia de este estudio radica en ser el primero en evaluar específicamente la disposición y las motivaciones para participar en investigación en personas diagnosticadas de TUS en nuestro medio. La mayoría de los participantes manifestaron su voluntad de participar en la investigación, siendo la proporción encontrada similar a la de otros estudios (Candilis et al., 2006). También se corresponde con el $30-40 \%$ de pérdidas que se suelen estimar en el cálculo de tamaños muestrales en investigaciones epidemiológicas (Marrugat, Vila, Pavesi y Sanz, 1999). No encontramos diferencias significativas entre población TUS y control en términos de disposición a participar ni tampoco entre los distintos subgrupos de sustancias consumidas.

Aproximadamente el $80 \%$ de la muestra (de las 205 personas a las que se les propuso participar, lo rechazaron 25), consintió participar en el estudio de aplicación de la entrevista MacCAT-CR. La proporción fue inferior, en torno al $75 \%$, cuando se propone el proyecto hipotético, con un riesgo superior al mínimo, al incorporar analíticas y efectos secundarios. Esta disminución en la disposición a participar permite comprobar cómo la percepción del riesgo influye en la participación en investigación. La diferencia entre ambos estudios podría ser mayor al advertir los 
sujetos un menor riesgo potencial del ensayo hipotético. Asimismo, el haber consentido previamente participar en nuestro estudio, podría haber aumentado la participación en el proyecto hipotético.

Uno de los elementos que más influyen en la participación en investigación es la confianza que deposita el sujeto en las personas que se lo proponen (Roberts, Warner, Anderson, Smithpeter y Rogers, 2004; Stroup et al., 2005). En el estudio CATIE realizado con la entrevista MacCAT-CR, el elemento predictor más importante de la disposición a participar fue el centro de referencia de los participantes. Ese resultado puede deberse al entrenamiento de los profesionales implicados y su relación previa con los entrevistados (Stroup et al., 2005). En nuestro caso, en los pacientes con TUS, los médicos responsables informaron sobre la posibilidad del estudio, lo que pudo contribuir a aumentar su participación. En los controles, la entrevistadora no era responsable directa de los pacientes ni pertenecía al centro de salud, aunque había rotado por el mismo. Aunque la confianza en las investigadoras fue un motivo poco esgrimido para participar en el estudio hipotético, sí que pudo influir en la aceptación de la aplicación de la entrevista MacCAT-CR (ver Figura 1).

La decisión de participar o no en el estudio hipotético se relacionó únicamente con el nivel cognitivo y el grado de alfabetización informática en los pacientes con TUS. No tenemos constancia de investigaciones previas sobre la influencia del grado de alfabetización informática para poder comparar nuestros resultados. Estos hallazgos tendrán que ser explorados en estudios venideros. En la bibliografía, la relación de la disposición a participar con el nivel cognitivo, la gravedad clínica y la capacidad de toma de decisiones es incierta, con resultados en ambos sentidos. Stanley y Stanley (1982), no encontró diferencias en cuanto a la decisión de participar o no según la gravedad clínica o el funcionamiento cognitivo. Sin embargo, Candilis (2006), sí encontró asociación entre la decisión de participar y la mayor capacidad para decidir según la escala MacCAT-CR, el mayor nivel educativo, la menor gravedad clínica y el menor deterioro cognitivo. Nuestro estudio, que concluye que no existe relación entre gravedad clínica, capacidad para tomar decisiones y disposición a participar, se une a la bibliografía existente sobre el tema que no refrenda dicha asociación.

La participación previa en investigación no se asoció a una mayor disposición a participar pero tampoco se identificó como una barrera. Por consiguiente, sujetos que nunca han sido reclutados para ensayos clínicos, podrían serlo si recibieran una información más adecuada. Estudios con grupos de usuarios (Fry, Madden, Brogan y Loff, 2006b), sugieren la utilidad de aclarar el beneficio potencial para los participantes en investigación e informarles más activamente del posible impacto de los resultados en el abordaje de su patología. Esto se contempla en los imperativos éticos del consentimiento informado y el principio de beneficencia (Beauchamp y Childress, 2009), aunque su aplicación requiere más énfasis (MacNeil y Fernández, 2006).

Los sujetos expresaron motivaciones para justificar su decisión de participar o no en el proyecto planteado concordantes con la bibliografía previa (Barrat et al., 2007; Candilis et al., 2006; Roberts et al., 2002). Las razones que se argumentaron a favor y en contra fueron apropiadas y se dedujeron lógicamente del estudio, tanto en población clínica como en la muestra control. El altruismo como principal motivo para participar, expresado como contribución a la ciencia, aparece en estudios realizados en población general y en diversos colectivos (Barrat et al., 2007; Candilis et al., 2006; Tromp et al., 2016).

La segunda razón para participar fue la posibilidad de obtener un beneficio personal. Aquí se encontraron respuestas relacionadas con la expectativa de un mejor tratamiento y con el mayor conocimiento de la enfermedad que también se argumentan en otros estudios (Candilis et al., 2006; Roberts et al., 2004). Se evidenciaron también elementos del error terapéutico, cuando la expectativa de obtener un beneficio pasa de percibirse como posibilidad a expresarse como convicción. Este elemento es muy importante a la hora de valorar la capacidad de toma de decisiones y se incluye en las preguntas de la entrevista MacCAT-CR. La división entre el razonable optimismo terapéutico y el convencimiento erróneo de que la participación comporta un bienestar personal, se rige por el grado de convencimiento (Jansen, 2006) que se valora de forma parecida a la evaluación de los trastornos del contenido del pensamiento. El error terapéutico aparece como elemento importante en nuestra muestra, como ocurre en otros estudios (Barrat et al., 2007; Tromp et al., 2016).

Tanto el altruismo como la posibilidad de un beneficio personal, se asociaron de forma univariada con la disposición a participar. Estos factores positivos pueden entenderse como incentivos potenciales para la participación en investigación en población TUS y ponen de relieve que tanto los beneficios potenciales como los reales de un estudio, son importantes para los posibles participantes.

Como ocurre también en otros estudios, algunos sujetos se refirieron a ambas motivaciones, altruistas y de potenciales beneficios personales, para decidir su participación (Candilis et al., 2006). Este hecho refleja la complejidad de valorar adecuadamente la apreciación de los sujetos de investigación, dado su carácter multideterminado.

En nuestro estudio no se hizo mención a los incentivos económicos, como motivación para participar en investigación. Puede haber ocurrido porque no se ofreció ninguna compensación por participar en el estudio inicial o porque en nuestro medio no esté extendida la idea de participar en investigación por dinero, como ocurre en otros contextos (Dunn et al., 2009). Existe controversia en torno a que los incentivos no sean sólo una compensación por el tiempo 
y los inconvenientes asociados con la participación , sino también inductores de la misma (Candilis et al., 2006; Misra et al., 2008). Hay datos que demuestran la relación entre la magnitud del incentivo y la modificación de la percepción del riesgo y del beneficio obtenido (Dunn et al., 2009). La investigación sobre los incentivos en pacientes TUS, ha intentado dar unas pautas prácticas en su aplicación desde una perspectiva ética, respetando el principio de justicia que permite la distribución equitativa de los beneficios de la investigación (Carter et al., 2012; Fry et al., 2006a).

Gran parte de los sujetos que se negaron a participar expresaron aversión a la investigación. En las respuestas encontramos términos peyorativos como ser utilizados como “conejillos de indias" que también aparecen muy frecuentemente en la bibliografía (Lebensburger et al., 2013). Este factor se mantiene en el análisis multivariante, por lo que se deberían concentrar los esfuerzos en tratar de combatir este limitador de la participación en investigación.

La valoración del riesgo de los efectos secundarios es muy variable, no obstaculizando la participación, si se consideran poco importantes y constituyendo una barrera para participar, si se les otorga mayor importancia. Según las leyes actuales (Real Decreto 1090/2015, 2015), para que un proyecto de investigación sea éticamente aceptable, el balance riesgo/beneficio para el participante debe ser adecuado. Aunque así sea, la valoración de los riesgos es diferente según las experiencias personales, culturales, e incluso por incentivos económicos. No obstante, una mejor información podría facilitar que el riesgo percibido se aproxime más al real (Mullin, 2002) y la evaluación de la comprensión y percepción de los riesgos podría ayudar a que el consentimiento sea realmente válido. Si tenemos en cuenta estos factores, podremos implementar de forma efectiva estrategias para mejorar el reclutamiento y adherencia en investigación clínica en población TUS.

\section{Limitaciones}

Debemos considerar unas limitaciones al interpretar nuestros resultados. En primer lugar, nuestro estudio se llevó a cabo en un entorno urbano con un número limitado de pacientes ambulatorios. Se necesitan investigaciones adicionales que evalúen nuestros resultados en otros entornos y participantes. Estudios con mayor tamaño muestral permitirían también un análisis de regresión múltiple con mayor número de variables.

Otra limitación a considerar es que la naturaleza no aleatoria de la muestra y la ausencia de otras sustancias (como heroína y ansiolíticos / hipnóticos) plantean cuestiones acerca de la generalización de los resultados. Estudios futuros deberían considerar estos aspectos.

Dado que los sujetos estaban considerando un fármaco hipotético para una patología que no era la suya, sería importante replicar estos hallazgos con medicamentos relacionados con su enfermedad.

\section{Conclusiones}

En este estudio, la disposición a participar de la población TUS fue similar a los controles. Un mayor nivel cognitivo y de alfabetización informática, eran más frecuentes entre los dispuestos a participar. Independientemente que decidieran participar o no, las razones esgrimidas eran adecuadas y concordantes con la literatura, aunque también se observaron elementos del error terapéutico en ambos grupos. Por lo tanto, las visiones negativas sobre las motivaciones de los TUS como participantes en investigación son infundadas. Identificamos unos factores predictivos de la disposición a participar a los que se deberían dirigir los esfuerzos para mejorar el reclutamiento.

\section{Reconocimientos}

No hubo ninguna fuente de financiación. Los autores agradecen a Da María Sánchez Muñoz y a D $D^{a}$ Beatriz Aguilera Sánchez, su colaboración en la extracción de la muestra. Agradecemos a los doctores Antonio Maurandi López y Guadalupe Ruiz Merino su asesoramiento estadístico. A todos los participantes del estudio les agradecemos su colaboración desinteresada.

\section{Conflicto de intereses}

Los autores no tienen conflictos de intereses que declarar.

\section{Referencias}

Aguayo, M. (2010). Cómo hacer una Regresión Logística con SPSS “ paso a paso” (I). Fundación Andaluza Beturia para la Investigación en Salud, 1-16.

American Psychiatric Association (2014). Diagnostic and Statistical Manual of Mental Disorders. (5th ed.). Washington DC: American Psychiatric Publishing.

Ammassari, A., Trotta, M. P., Murri, R., Castelli, F., Narciso, P., Noto P.,... AdICoNA Study Group. (2002). Correlates and predictors of adherence to highly active antiretroviral therapy: Overview of published literature. Journal of Acquired Immune Deficiency Syndrome, 31, 123-127.

Appelbaum, P. S. y Grisso, T. (2001). MacCAT-CR: MacArthur Competence Assessment Tool for Clinical Research. Professional. Sarasota, FL: Professional Resource Press.

Baón, B. S. (2013). Adaptación y Validación Española de la Entrevista Macarthur Competency Assessment Tool for Clinical Research (MacCAT-CR) y de un Cuestionario Breve para Evaluar la Capacidad de las Personas para Consentir Participar en Investigación. Tesis Doctoral en Formato Electrónico. Madrid: Repositorio Institucional E-Prints Universidad Complutense.

Barrat, M. J., Norman, J. S. y Fry C. L. (2007). Positive and negative aspects of participation in illicit drug research: Implications for recruitment and ethical conduct. Inter- 
national Journal of Drug Policy, 18, 235-238. doi:10.1016/j. drugpo.2006.07.001.

Beauchamp, T. L. y Childress, J. F. (2009). Principles of Biomedical Ethics. (6th ed.). New York: Oxford University Press.

Brintnall-Karabelas, J., Sung, S, Cadman, M. E., Squires, C., Whorton, K. y Pao, M. (2011). Improving Recruitment in Clinical Trials: Why Eligible Participants Decline. Journal of Empirical Research on Human Research Ethics, 6, 69-74. doi:10.1525/jer.2011.6.1.69.

Candilis, P. J., Geppert, C. M., Fletcher, K. E., Lidz, C. W. y Appelbau, P. S. (2006). Willingness of subjects with thought disorder to participate in research. Schizophrenia Bulletin, 32,159-165. doi:10.1093/schbul/sbj016.

Carter, A. y Hall, W. (2012). Ethics of addiction research. En Edwards, G. (Ed.), Addiction Neuroethics. The Promises and Perils of Neuroscience Research on Addiction (pp. 146160). New York: Cambridge University Press.

Dunn, L. B., Kim, D. S., Fellows, I. E. y Palmer, B. W. (2009). Worth the risk? Relationships of incentives to risk and benefit perceptions and willingness to participate in schizophrenia research. Schizophrenia Bulletin, 35, 730737. doi:10.1093/schbul/sbn003.

Endicott, J., Spitzer, R. L., Fleis, J. L. y Cohen, J. (1976). The Global Assessment Scale: A procedure for measuring overall Severity of Psychiatric disturbance. Archives of General Psychiatry, 33, 766-771.

Fry, C. L. y Dwyer, R. (2001). For love or money? An exploratory study of why injecting drug users participate in research. Addiction, 96, 1319-1325. doi:10.1080/09652140120070373.

Fry, C. L., Hall, W., Ritter, A. y Jenkinson, R. (2006a). The Ethics of Paying Drug Users Who Participate in Research: a Review and Practical Recommendations. Research Subject Payment Ethics, 1, 21-36. doi:10.1525/ jer.2006.1.4.21.

Fry, C. L., Madden, A., Brogan, D. y Loff, B. (2006b). Australian resources for ethical participatory processes in public health research. Journal of Medical Ethics, 32, 186. doi:10.1136/jme.2005.013243.

Geppert, C. M., Candilis, P. J., Baker, S., Lidz, C. W. y Appelbaum, P. S. (2014). Motivations of Patients with Diabetes to Participate in Research. AJOB Empirical Bioethics, 5, 1421. doi:10.2105/AJPH.2014.301881.

Guy, W. (1976). Clinical Global Impression Scale. En Guy, W. (Ed.), ECDEU Assessment Manual for Psychopharmacology (pp. 217-223). Rockville: Department of Health, Education, and Welfare Public Health Service Alcohol, Drug Abuse, and Mental Health Administration.

Jansen, L. A. (2006). The Problem with Optimism in Clinical Trials. IRB: Ethics Eீ Human Research, 28, 13-19.

Lawton, J., Snowdon, C., Morrow, S., Norman, J. E., Denison, F. C. y Hallowell, N. (2016). Recruiting and consenting into a peripartum trial in an emergency setting: a qualitative study of the experiences and views of women and healthcare professionals. Trials, 17, 1-14. doi:10.1186/s13063-016-1323-3.

Lebensburger, J. D., Sidonio, R.F., DeBaun, M. R., Saffor, M. M., Howard, T. H. y Scarinci, I. C.(2013). Exploring Barriers and Facilitators to Clinical Trial Enrollment in the Context of Sickle Cell Anemia and Hydroxyurea. Pediatric Blood E Cancer, 60, 1333-1337. doi:10.1002/ pbc.24486.

Lobo, A., Saz, P., Marcos, G., Día, J. L., de la Cámara, C., Ventura, T.,... Aznar, S. (1999). Revalidation and standardization of the cognition mini-exam (first Spanish version of the Mini-Mental Status Examination) in the general geriatric population. Medicina Clínica, 112, 767774.

MacNeil, S. D. y Fernández, C. V. (2006). Informing research participants of research results: Analysis of Canadian university based research ethics board policies. Journal of Medical Ethics, 32, 49-54. doi:10.1136/ jme.2004.010629.

Marrugat, J., Vila, J., Pavesi, M. y Sanz F. (1999). Estimación del tamaño de la muestra en la investigación clínica y epidemiológica. Medicina Clínica, 111, 267-276.

Misra, S., Socherman, R., Park, B. S., Hauser, P. y Ganzini, L. (2008). Influence of mood state on capacity to consent to research in patients with bipolar disorder. Bipolar Disorder, 10, 303-309. doi:10.1111/j.13995618.2007.00525.x.

Morán-Sánchez, I., Luna, A., Sánchez, M., Aguilera, B. y Pérez-Cárceles, M. D. (2016). Decision-making Capacity for Research Participation among Addicted People: a cross-sectional study. BMC Medical Ethics, 17, 1-10. doi:10.1186/s12910-015-0086-9.

Morera, B. (2000). Aspectos bioéticos de la asistencia al drogodependiente. Adicciones, 12, 515-526.

Mullin, S. (2002). Communicating risk: closing the gap between perception and reality. Journal of Urban Health, 79, 296-297. doi:10.193/jurban/79.3.296.

Nogué, S. y Miró, O. (2015). Núcleos y ámbitos de investigación sobre adicciones: necesidad de una visión más amplia. Adicciones, 27, 75-76.

Nuñez, E., Steyerberg, E. W. y Nuñez, J. (2011). Regression modeling strategies. Revista Española de Cardiología, 64, 501-507. doi:10.1016/j.recesp.2011.01.019.

Ortega, M. y Cayuela, A. (2002). Regresión logística no condicionada y tamaño de muestra: una revisión bibliográfica. Revista Española de Salud Pública, 76, 85-93.

Real Decreto 1090/2015, de 4 de diciembre por el que se regulan los ensayos clínicos con medicamentos, los Comités de Ética de la Investigación con medicamentos y el Registro Español de Estudios Clínicos. Boletín Oficial del Estado. Madrid, 24 de diciembre de 2015, núm.307, pp. 121923-121964.

Roberts, L. W., Warner, T. D., Anderson, C. T., Smithpeter, M. V. y Rogers, M. K. (2004). Schizophrenia research partici- 
pants' responses to protocol safeguards: recruitment, consent, and debriefing. Schizophrenia Research, 67, 283-291.

Roberts, L. W., Warner, T. D., Brody, J. L., Roberts, B., Lauriello, J. y Lyketsos, C. (2002). Patient and Psychiatrist Ratings of Hypothetical Schizophrenia Research Protocols: Assessment of Harm Potential and Factors Influencing Participation Decisions. American Journal of Psychiatry, 159, 573-584.

Seelig, B. J. y Dobelle, W. H. (2001). Altruism and the Volunteer: Psychological Benefits from Participating as a Research Subject. ASAIO Journal, 47, 3-5.

Stanley, B. y Stanley, M. (1982). Testing competency in psychiatric patients. IRB, 4, 1-6.

Stroup, T. S., Appelbaum, P. S., Swartz, M. S., Patel, M., Davis, S., Jeste, D. V.,... Lieberman, J.(2005). Decision-making capacity for research participation among individuals in the CATIE schizophrenia trial. Schizophrenia Research, 80, 1-8. doi:10.1016/j.schres.2005.08.007.

Tromp, K., Zwaan, C. M. y van de Vathorst, S. (2016). Motivations of children and their parents to participate in drug research: a systematic review. European Journal of Pediatrics, 175, 599-612. doi:10.1007/s00431-016-2715-9. 